\title{
QS能
}

Carlota Lorenzo Romero*

Juan Antonio Mondéjar Jiménez**

Leticia del Pozo Ruiz*

\section{PARTICIPACIÓN DEL CONSUMIDOR ONLINE EN EL SECTOR TEXTIL}

Los canales de distribución online son significativas plataformas de venta que ofrecen la posibilidad de aplicar estrategias de colaboración e innovación entre clientes y marcas. El objetivo del presente documento es destacar a través de una revisión de la literatura, la continua transformación online en la industria textil a través de acciones de interacción más personalizadas por parte de las marcas textiles. Con la finalidad de poder satisfacer la exigente demanda de los actuales consumidores en el sector textil, la constante evolución en la industria de la moda también es percibida a través de planteamientos que aportan un nuevo valor al consumidor final, como la cocreación online. Situando actualmente al consumidor final, también a través de estrategias multicanal, como un agente clave en los procesos de creación y decisión.

Palabras clave: cocreación en moda, experiencia de cocreación, distribución online, multicanalidad. Clasificación JEL: L1, L11, L170, M19.

\section{Introducción}

La participación de los consumidores en acciones de colaboración online con marcas textiles define un nuevo escenario de actuación conjunta a través de las múltiples opciones que ofrece un canal como Internet. Empresas de diversos sectores enfocan actualmente una interacción de forma online entre la marca y el

\footnotetext{
* Facultad de Ciencias Económicas y Empresariales, Universidad de Castilla-La Mancha.

** Facultad de Ciencias Sociales, Universidad de Castilla-La Mancha.

Versión de junio de 2018.

El presente trabajo se enmarca dentro del Proyecto de Investigación en curso titulado: La colaboración abierta en Internet como estrategia de innovación e internacionalización del sector de moda y complementos (2015-2018), otorgado por la Fundación Ramón Areces.
}

cliente basada en el individuo como elemento clave, como por ejemplo a través de las comunidades online que fomentan la comunicación, la participación y la compra (Chae y Ko, 2016). La elevada competencia global ha ido cambiado la manera de introducir nuevos productos y servicios en el mercado, analizando los diferentes segmentos de cliente (Hu, Lin, Qian y Sun, 2018) o aplicando estrategias que, basadas en la tecnología, gestionan la relación con el cliente, el comportamiento del consumidor, las comunidades de marca online o las redes sociales (Gruner, Homburg y Lukas, 2014; Hinz, Skiera, Barrot y Becker, 2011). Anteriormente, se planteaba una interacción empresarial con el cliente basada en el producto, el consumidor y el contexto de la $\square$ 
ventas (Kasof, 1995), pero ese enfoque tradicional se ha transformado en acciones más directas y centradas en el consumidor, permitiendo que la experiencia del cliente sea ahora mucho más personalizada y satisfactoria (Hosany y Witham, 2010; Rubera, Ordanini y Mazursky, 2010; Tsiotsou y Ratten, 2010). En base a las actuales tendencias de comunicación, Internet se configura como una excelente plataforma en la que cada vez más empresas confían para ofrecer diversas herramientas de interacción con los clientes, de forma más personalizada (Leeflang, Verhoef, Dahlström y Freundt, 2014). De esta forma, Internet es una plataforma capaz de elevar el valor de la marca a través del intercambio de ideas o de la transmisión de información entre personas, de forma online (Kim y Ko, 2012). Las opciones de interacción online entre clientes y marcas también dentro del sector textil no solo brindan al consumidor la oportunidad de generar ideas, sugerencias, reflexiones o experiencias, sino que, además, permiten a las empresas de la industria textil aplicar estrategias online con menor inversión económica. De forma general, los proyectos con enfoques altamente creativos se basan en motivaciones muy diversas, que pueden abarcar perspectivas culturales, sociales o económicas (Ordanini, Miceli, Pizzetti y Parasuraman, 2011). La innovación en ámbitos empresariales se enfoca principalmente en obtener el máximo crecimiento a través de acciones diferenciadoras, consideradas como un factor de éxito en las organizaciones que operan en mercados altamente competitivos (Naranjo-Valencia, Jiménez-Jiménez y SanzValle, 2016). Por ello, las empresas que aplican estrategias innovadoras de forma online son más proclives a obtener mayor rentabilidad a través de acciones que aporten innovación al consumidor. Respecto a las fuertes demandas de los consumidores actuales o a su percepción sobre las características que deben cumplir las tiendas para satisfacer mejor las demandas del cliente (Goodman y Remaud, 2015; Im, Montoya y Workman, 2012; Maruyama y $\mathrm{Wu}, 2014)$, las marcas efectúan acciones en ambientes de mercado caracterizados por continuos cambios técnicos o de incertidumbre. Por ello, la innovación o aquellas estrategias que la incluyan son consideradas fundamentales para obtener la máxima competitividad y diferenciación entre empresas de cualquier sector (Damanpour y Gopalakrishnan, 2001). En la industria de la moda, los elementos sensoriales son altamente relevantes porque los consumidores buscan durante sus procesos de compra factores como la atracción visual del producto o el entretenimiento. De modo que las marcas textiles enfocan sus estrategias hacia la obtención de una experiencia de cliente única e irrepetible (Eroglu, Machleit y Davis, 2003). Las nuevas tecnologías facilitan ampliamente este proceso de satisfacción de experiencias, ya que permiten generar, incluso, una experiencia integrada entre la tienda física y la tienda online. El actual comercio retail o minorista concentra diferentes canales con el objetivo de mejorar la experiencia del consumidor a través de estrategias de venta más atractivas. En los actuales canales de venta al por menor se aprecia una evolución desde la multicanalidad, basada en la venta a través de varios canales, pero de forma independiente, hacia estrategias de omnicanalidad, centradas en la venta a través de varios canales simultáneos pero con un trato unificado hacia el cliente en cada uno de ellos. La evolución de la multicanalidad hacia la omnicanalidad en la venta al por menor (Verhoef, Kannan e Inman, 2015) se aprecia en acciones de consumo como la recogida de un producto textil en tienda física mediante $\triangleright$ 
previa compra online, o de estrategias de tecnología avanzada, como la presencia de espacios interactivos en tiendas físicas para la elección de prendas, conectados con las redes sociales (Blázquez, 2014). Además, como destacaron autores como Chen, Shang y Kao, (2009), factores como la actitud o el control percibido inciden de forma relevante sobre el comportamiento del consumidor en su experiencia de compra, en la fase postcompra o en las decisiones que pueden motivar a los consumidores a repetir sus experiencias de compra o participación, en canales electrónicos. Resulta necesario que las compañías ofrezcan herramientas de participación al cliente, para que su experiencia de compra pueda transformarse en algo único, agradable y diferenciador (Chen, Shang y Kao, 2009; Sicilia y Ruiz, 2010), evitando, gracias a ello, que los consumidores puedan experimentar emociones negativas durante sus procesos de compra o de participación online. De igual forma, las marcas textiles se mantienen constantes en establecer el máximo contacto entre el consumidor y la tecnología para obtener mejoras en la experiencia del cliente tanto en tiendas físicas como en tiendas online (Ono et al., 2012).

\section{Desarrollo}

\subsection{Nuevo marco online en el sector de la moda en España}

La moda y su relación con la comunicación es un planteamiento cada vez más desarrollado en las investigaciones actuales (Granata, 2012; McNeil, 2010; Tseëlon, 2010), pero que, por las variables, características y diferentes formas de expresión que conforman este sector, todavía plantea dificultades para poder delimitar estudios más significativos respecto a esta relación. Para autores como Torregrosa, Noguera, Sánchez-Blanco y del Río (2014), la moda es un concepto que enmarca diversas perspectivas, donde destacan claramente tres dimensiones: dimensión antropológica (es una realidad que se apoya en la naturaleza corporal de cada persona), dimensión económica (ofrece la posibilidad de vender productos a través de la innovación estética) y dimensión cultural (está compuesta de un contexto altamente creativo y perdurable). No obstante, a pesar de su importancia como concepto en sí mismo, la industria de la moda no tendría tal visibilidad y relevancia entre los consumidores del mercado y en la sociedad actual sin la difusión otorgada durante décadas por los medios de comunicación masivos (Jackson y Shaw, 2006; Kuruk, 2008). A esta perspectiva se unen actualmente los canales de comunicación online, que no solo permiten la distribución de las prendas textiles de forma global, sino que también son excelentes plataformas para compartir información y establecer una mayor interacción entre los consumidores y las marcas textiles. La importancia de aplicar herramientas online que puedan determinar cambios conductuales de los consumidores en base a su participación y a su percepción de valor reside en la interacción, el diálogo y la aportación, como factores fundamentales para obtener mejores resultados de forma conjunta entre consumidores y empresas (Sánchez, Mieres y Vijande, 2013).

La moda conforma un sector en relación directa con la innovación, los cambios sociales o culturales, la transformación y el crecimiento económico, teniendo el sector textil una estrategia diferenciadora en un canal de distribución como Internet. Numerosas marcas de moda buscan construir y mantener relaciones de $D$ 
lealtad con sus clientes a través de Internet y de herramientas como los dispositivos móviles, donde la participación del cliente es instantánea y requiere una implicación muy elevada, siendo posible personalizarla al máximo (Carlson, O'Cass y Ahrholdt, 2015; Chae, Ko y Han, 2015). Esta nueva situación se refuerza gracias a plataformas como los blogs o las redes sociales, donde el usuario o cliente online se identifica con el contenido expuesto por la empresa de moda, siguiendo a la marca de forma online, opinando o aconsejando a otros usuarios o clientes sobre su propia experiencia o convirtiendo la simple acción de pulsar el botón «me gusta» en una potente herramienta, aportando valiosa información para futuras estrategias comerciales (Llorente, Bartolomé y Navarro, 2013). Gracias a las redes sociales se establece una comunicación mucho más directa con el consumidor actual y las marcas pueden conocer de forma más detallada las preferencias o las exigencias de cada uno. Para ello, es necesario que los clientes aporten información a las compañías de forma previa, y cada vez más marcas ofrecen a los usuarios la posibilidad de crear, participar o debatir de forma online a través de estas plataformas, con la finalidad de redirigir la información obtenida hacia nuevas planificaciones de negocio enfocadas al mayor beneficio económico (Varadarajan y Yadav, 2010). Este tipo de estrategias vienen precedidas por la situación actual de un mercado global donde cada vez existen clientes más complejos y con mayor diversidad de necesidades de consumo, pero más interconectados (Gambeti y Graffigna, 2011). El hecho de que los consumidores participen de forma activa en un nuevo marco online permite una generación de experiencias que los clientes identificarán de forma directa con las marcas adquiriendo el denominado brand engagement (Hollebeek,
2011). En este nuevo contexto online, en el que el sector textil cuenta con activa presencia, las empresas se esfuerzan en conseguir que los consumidores identifiquen las marcas de cada sector como modelos de confianza con los que participar y colaborar de forma online. Marcas españolas como Grupo Inditex, Camper, Mango, Bimba y Lola o Grupo Cortefiel cuidan al máximo el contenido de sus páginas web y de sus perfiles corporativos en redes sociales. Incluso aplican estrategias online asignando perfiles destacados en plataformas como Instagram a personas influyentes en el sector de la moda en España, denominados influencers de moda. La gran influencia de Internet en el contexto social y en sectores como la moda ha transformado de tal modo las relaciones de comunicación y de consumo que los nuevos líderes de opinión en un canal online se han convertido en elementos fundamentales en los procesos de comunicación con el receptor (Sádaba y San Miguel, 2014).

La finalidad de las marcas minoristas en todo este proceso se orienta en generar relaciones a largo plazo con los usuarios o clientes online, creando una relación más personal con el consumidor para obtener el máximo beneficio empresarial (Del Pino, Galán, Castelló y Ramos, 2014). Respecto a la compra a través del canal online en España, según datos aportados por el último estudio anual de ecommerce elaborado por IAB Spain (2017), un 73 por 100 del total de la población internauta española compró de forma online en 2017 , suponiendo una cifra de 16,4 millones de compradores online y siendo el 98 por 100 de estos usuarios de alguna red social. Respecto a los usos y hábitos de compra online en España durante 2017, destacó la preferencia para productos físicos (92 por 100), como el caso de las prendas textiles, respecto a la compra de servicios (83 por $\triangleright$ 
100). La moda, el calzado y los complementos se establecieron como preferencias de compra de los consumidores online (60 por 100), precedidas ligeramente por viajes (75 por 100), ocio y cultura (72 por 100) o tecnología (67 por 100). Respecto al lugar de adquisición de la compra, el mismo informe IAB Spain (2017) indica que el 87 por 100 de los internautas españoles prefirió adquirir los productos en tiendas con venta únicamente online (destacando como factores positivos la comodidad, la lejanía de la tiendas físicas o la disponibilidad horaria del usuario para la compra), frente al 73 por 100, que prefirió la compra en aquellas tiendas que vendiesen de forma combinada, online y física. Igualmente en 2017, el 95 por 100 de los compradores online en España realizaron sus compras mediante el ordenador, frente al 37 por 100 que las realizaron a través de sus teléfonos móviles. Respecto a los motivos por los que los usuarios españoles optan por realizar sus compras de forma online, un 98 por 100 respondió a motivos de conveniencia; un 97 por 100 a motivos basados en la amplia variedad de los productos en este canal; un 93 por 100 a motivos relativos al precio; un 74 por 100 por la confianza de compra respecto al canal online; un 49 por 100 por recomendación de terceros y un 31 por 100 debido al impacto de la publicidad online.

En el sector textil, las plataformas online otorgan oportunidades altamente competitivas y diferenciadoras, basadas en la comunicación, la difusión y la comercialización de las prendas y los complementos textiles de forma mucho más eficaz. El Informe de la moda online en España (2018), destaca la relevante importancia de Internet en el sector de la moda, siendo cada vez más significativa la compra de moda a través del canal online y suponiendo durante el año 2017 una cuota de mercado del
5,4 por 100 sobre el total de las ventas de moda en España. Respecto al total de ventas de moda online en España en 2017, el calzado supuso un 7,5 por 100, por delante del 5,6 por 100 para los accesorios, del 5,2 por 100 para el textil de hogar y del 4,7 por 100 para las prendas de vestir. Dentro del mismo Informe de la moda online en España (2018), y según la información de la Comisión Nacional de los Mercados y la Competencia (CNMC), se expone un evolutivo en las ventas de moda online entre los años 2012 y 2017. Según los datos de CNMC (2018), hasta el tercer trimestre de 2017 el beneficio obtenido en venta fue de 1.658,9 millones de euros, el cual contrasta de forma significativa con el beneficio obtenido en el año 2013 (773,7 millones de euros) y en el año 2015 (1.766,4 millones de euros), superados únicamente todos ellos por el mejor registro en ventas de moda online en España hasta el momento, obtenido en el año 2016 y correspondiente a una cifra de 2.015,2 millones de euros. En referencia a las ventas online de las prendas de vestir en España, según la CNMC (2018), hasta el tercer trimestre del año 2017 el beneficio obtenido fue de 1.215,7 millones de euros, en comparación por ejemplo con el año 2014 , cuando supuso una cifra de 527,5 millones de euros. No obstante, el máximo valor fue alcanzado en el año 2016 con 1.460,1 millones de euros. Respecto al calzado y artículos de cuero, igualmente la cifra máxima de venta fue obtenida en el año 2016, correspondiendo a 272,2 millones de euros, por encima de los 226,5 millones de euros registrados en el año 2017. Los consumidores online son ampliamente diversos y cambiantes y este factor exige también a las empresas del sector textil una constante transformación para la obtención de clientes a través de plataformas de venta online que apliquen estrategias diferenciadoras. Se $\triangleright$ 
observa una evolución respecto al tradicional planteamiento sobre la venta de la moda, la comunicación y el consumidor en España con la gestión de actuales espacios online que permiten al público aportar sus opiniones sobre las marcas, compartir información de forma más activa y comunicarse de forma más global, a través de múltiples acciones planteadas en el canal online (Pereira, García, González y Contreras-Espinosa, 2014).

\subsection{Importancia de las acciones de cocreación}

Los procesos de producción, distribución y consumo se han ido modificando con la necesidad de ir adaptando los productos y servicios hacia un nuevo mercado globalizado en el que los consumidores son cada vez más exigentes y complejos. La evolución del mercado ha generado que el valor sea considerado como un activo relevante, convirtiendo con ello a las marcas en elementos fundamentales de propuestas de creación de valor e interviniendo éstas de forma directa en los niveles de confianza y de fidelidad de los clientes. Como indican diversos autores (Prahalad y Ramaswamy, 2000; Vargo y Lusch, 2004; Von Hippel, 2005), las empresas potencian sus estrategias corporativas y a través de acciones de participación conjunta con los clientes, en las que estos adoptan un rol más participativo desarrollando nuevos productos $y$ servicios y otorgando amplios beneficios a las corporaciones. En este contexto de intercambio y de forma genérica, el concepto de cocreación es expuesto por diversos autores (Bason, 2010; Prahalad y Ramaswamy, 2004) como un proceso de creación de nuevas ideas, diseñadas a través de la participación conjunta y evolutiva entre diversos agentes involucrados (clientes, públicos objetivos, empresas, etcétera) en procesos de creación y desarrollo de nuevos productos o servicios y con la finalidad de generar una aportación más significativa de valor conjunto. La cocreación se configura como una actividad de participación en la creación de valor entre varias partes implicadas, a través de una participación impulsada por motivaciones personales o propuestas externas, pero orientada a colaborar, experimentar, diseñar o dialogar con la organización (Prahalad y Ramaswamy, 2004; Sawhney, Verona y Prandelli, 2005; Vargo y Lusch, 2004). La adecuada gestión de las acciones de cocreación deriva en ventajas competitivas para las organizaciones, tales como mayor productividad y reducción de costes de producción y mejor eficiencia e innovación en los procesos de desarrollo de los productos y servicios (Hull, 2004; Payne, Storbacka y Frow, 2008; Prahalad y Ramaswamy, 2000; Sánchez et al., 2013; Vargo y Lusch, 2004). Las acciones de cocreación suponen, de igual forma, nuevas herramientas capaces de diseñar o configurar entre las empresas y consumidores ambientes de experiencia, aportando beneficios mutuos para ambas partes (Ramaswamy y Gouillart, 2010). Actualmente, los consumidores interactúan con las marcas de forma más directa y natural, dentro de procesos propuestos por las compañías, con una integración entre las partes que comprende varios canales y donde el objetivo es desarrollar la experiencia de cliente de forma integral y más satisfactoria (Lemon y Verhoef, 2016). A pesar de que las actividades de creación en sí se han desarrollado con un largo enfoque en el tiempo, identificar el concepto de cocreación en la literatura referente al marketing es relativamente novedoso (Grönroos, Strandvik y Heinonen, 2015; Ind y Coates, 2013), ya que las actuales formas de comunicación entre empresas y consumidores han originado $D$ 
nuevas acciones de creación de valor donde los consumidores participan ahora de forma más activa con las empresas del mercado (Saarijärvi, Kannan y Kuusela, 2013). Igualmente, las acciones de cocreación se identifican con creaciones de aportación de valor desarrolladas a través de interacciones que otorgan al individuo la posibilidad de aportar y participar, obteniendo experiencias personalizadas (Prahalad y Ramaswamy, 2004). Otros autores (Grönroos et al., 2015; Neghina, Caniëls, Bloemer y Van Birgelen, 2015; Vargo y Lusch, 2011) también indican que las acciones de cocreación pueden implicar más o menos agentes (consumidores, organizaciones, proveedores, etcétera), pero que, de cualquier forma, se produce una interacción conjunta entre todos ellos, obteniendo como resultado un proceso creativo, interactivo y social para crear valor gracias a la participación y aportación compartida (Roser, Defillippi y Samson, 2013).

La importancia de que exista conexión entre las empresas y los consumidores se refuerza, más si cabe, con el actual rol activo del consumidor para crear valor. El consumidor ya no actúa únicamente de forma pasiva ante lo que la empresa pueda ofrecerle, sino que a través de acciones de cocreación es mucho más participativo y más activo en sus decisiones, aportando, configurando o rediseñando productos o servicios (Prahalad y Ramaswamy, 2004). Este planteamiento se integra actualmente en comunidades virtuales, foros de discusión online o plataformas online donde los consumidores y usuarios pueden exponer sus opiniones, sugerencias, quejas o ideas respecto a los productos o servicios que comercializan las empresas. De esta forma, las compañías entienden la importancia de fomentar el valor con el consumidor, de forma unida y personalizada (Prahalad y Ramaswamy, 2000 y 2004). En este aspecto, las organizaciones proponen procesos externos de creación, innovación o desarrollo a sus diferentes públicos, alejados de los anteriores procesos de I+D, que estaban realizados únicamente dentro de la organización. Las empresas aplican ahora estrategias que les permiten integrar opciones y recursos con los consumidores del mercado, con otros agentes externos o con la sociedad, con el objetivo de obtener acciones de valor conjunto (Bettencourt, Lusch y Vargo, 2014). Por ello, las acciones de cocreación de valor son muy relevantes respecto a la transferencia de información y comunicación, integrando, gracias a ellas, recursos para generar un beneficio mutuo respecto a factores como capacidades, necesidades, conocimiento o técnicas (Ballantyne y Varey, 2008; Gummesson y Mele, 2010). Por ello, las organizaciones que buscan diferenciación respecto a sus competidores del mercado deberían aplicar sus estrategias hacia acciones de valor conjunto más personalizadas con el consumidor para poder obtener nuevos beneficios y procesos de interacción mucho más satisfactorios (Grönroos y Voima, 2013; Prahalad y Ramaswamy, 2004).

\subsection{El consumidor como cocreador en el entorno online}

Con base en la importancia que adopta el consumidor en todo este proceso, cabe resaltar que aquel es un agente clave en la relación establecida entre la creación, el marketing y el consumo, ya que a través de sus aportaciones hacia los productos o servicios es capaz de crear valor autónomamente (Grönroos y Voima, 2013). De igual forma, el comportamiento altamente activo y participativo del consumidor actual posibilita las acciones de cocreación con las empresas (Yi y Gong, 2013), ya que la $\triangle$ 
actitud para participar en la cocreación se conforma por diversos factores. Entre ellos se puede encontrar el grado de intención del usuario de participar en acciones de cocreación online (Constantinides, Brünink y Lorenzo-Romero, 2015) o incluso las diferentes dimensiones de experiencia de cocreación que puede obtener el usuario cocreador con este proceso (Verleye, 2015).

Varios estudios destacan la importancia de analizar el comportamiento del consumidor en los procesos de cocreación de valor (Vega, Revilla y Cossío, 2013; Yi y Gong, 2013). En mercados cada vez más cambiantes y competitivos, como el del sector textil, la participación activa de los consumidores con las marcas y los nuevos roles de los consumidores como cocreadores representan una ventaja diferencial para las empresas. Desde una perspectiva tradicional, el diseño o desarrollo de productos y servicios ha sido considerado un proceso interno dentro de las organizaciones. Sin embargo, esa configuración se ha transformado actualmente, otorgando al consumidor un rol más activo, apareciendo una tipología de consumidor-creador hacia los productos y servicios que compra o utiliza activamente (O’Hern y Rindfleisch, 2010). Según Ramaswamy y Gouillart (2010), el nuevo concepto de consumidor se diferencia del concepto tradicional, ya que ahora existe un compromiso personal del individuo con el valor de la cocreación. Grönroos y Voima (2013) indican que los negocios actuales deben fundamentarse en las creaciones recíprocas de valor, dejando en un segundo plano el producto o el servicio en sí para enfocarse en las transmisiones de diálogo y de aportación conjunta a través de métodos de cocreación. $Y$ para autores como Heinonen y Strandvik (2015), la clave de las acciones de cocreación de valor se encuentra en las intenciones, motivaciones y acciones de cada consumidor, así como en el valor que los consumidores perciben y que posteriormente aportan. Respecto a los motivos por los que los individuos participan en comunidades online y en acciones de cocreación como las efectuadas en el sector textil, una de las principales investigaciones centradas en ello fue expuesta por los autores Wasko y Faraj (2000), indicando que existen factores motivadores que incitan a participar en acciones de cocreación, como el propio interés del usuario en desarrollar una actividad novedosa o su previa relación directa con una comunidad online. Otros autores expusieron de forma posterior (Ahearne, Bhattacharya y Gruen, 2005) que los consumidores se identifican con las empresas de forma personal, lo cual les proporciona querer participar, colaborar o aportar en este tipo de procesos. Desde otro enfoque más actual, Fuchs y Schreier (2011) indican que los consumidores tienen un amplio poder de decisión y son más proclives a este tipo de acciones comportamentales, gracias, también, a la gran interacción que ofrecen las empresas con el cliente. Autores como Füller, Hutter y Faullant (2011) destacaron la importancia de cómo los consumidores que participan en acciones de cocreación online perciben un mayor compromiso o engagement con las marcas, ya que a través de la participación en este tipo de acciones el consumidor asume un rol de poder definido por su experiencia individual, autodeterminación o eficacia que conduce hacia una mayor confianza sobre la marca concreta con la que realiza las acciones de cocreación.

\subsection{Experiencia del consumidor en cocreación textil}

Diversos autores (Hoyer, Chandy, Dorotic, Krafft y Singh, 2010; Nambisan y Baron, 2009; Verleye 2015) han planteado interesantes $D$ 
estudios sobre los factores motivacionales de los usuarios para participar en acciones de cocreación online, destacando la importancia motivacional de los individuos en este tipo de procesos. El sector textil es un claro reflejo de la imagen externa de cada individuo, por lo que las opiniones o sugerencias sobre las marcas o las tendencias de moda ganan relevancia en una esfera online, donde la información es compartida de forma muy amplia. Como señalan autores como Vallejo, Redondo y Acerete (2015), una práctica común en las empresas es incorporar, en sus propias plataformas de venta online, espacios donde los usuarios puedan compartir información sobre los productos y servicios ofertados para generar un bocaoído electrónico (eWom) entre consumidores. Esta información supone igualmente una relevante base de datos para las empresas del sector textil, ya que les permite poder configurar mejor las necesidades del mercado. Según exponen Del Olmo y Gascón (2014), las empresas del sector de la moda pueden aproximarse al cliente de una forma diferente gracias al entorno online, ofreciéndole alternativas para la creación y la difusión a través de herramientas colaborativas, donde en numerosas ocasiones el proceso ya no corresponde a la empresa en sí, sino que los consumidores son los que gestionan toda la información a través de espacios donde indicar sus opiniones o su grado de satisfacción personal con la marca textil. Los mismos autores indican que las empresas textiles involucran a los usuarios online consiguiendo que participen libremente en procesos de cocreación donde diseñan, comparten su experiencia, recomiendan o disuaden sobre las prendas del mercado. Exponen el ejemplo de la marca de calzado deportivo Munich, que ofrece a través de la aplicación Munich my Way la opción de diseño y posterior obtención del producto. También, el ejemplo de plataformas como eYeka.com, donde las empresas pueden organizar iniciativas libres de cocreación online para los usuarios.

Respecto a las marcas españolas del sector textil y complementos que ofrecen la posibilidad de cocreación al usuario de forma online, podemos indicar que el Grupo Cadarso, a través de su marca WatxandCo, permite al usuario diseñar íntegramente y comprar de forma posterior un modelo de reloj. La empresa LACAMBRA ofrece la opción de un diseño personalizado por el usuario y de posterior compra de bolsos de piel, con multitud de opciones para su creación. La marca con producción madrileña Bálamor permite al usuario diseñar bailarinas de forma personalizada con amplia variedad de colores o diversos materiales; o la marca La Pajarera de Madera, con talleres en Salamanca y Zaragoza, ofrece la posibilidad de diseñar y comprar pajaritas de madera personalizadas. Igualmente, el diario español de información deportiva Marca, a través de un configurador instalado en su propia página web que contaba con acceso a redes sociales para su posterior publicación, facilitó a los usuarios la opción de diseñar una camiseta personalizada de cualquier equipo de Liga Española de Fútbol para la temporada 2016/17. Por ello, las acciones de cocreación contribuyen a la productividad y la eficiencia en las corporaciones, obteniendo de esta forma, aportaciones y esfuerzos de los consumidores a través de una participación activa y ampliamente colaborativa (Magnusson y Kristensson, 2003). Con base en la experiencia del consumidor, cuando interactúa con las empresas participando en este tipo de procesos colaborativos, resulta relevante destacar su importancia, guiando a las empresas hacia las estrategias que considera más apreciadas o hacia aquellas que serían $\triangleright$ 
deseadas por su parte gracias a su propia experiencia. Para Füller et al. (2011), la importancia de identificar experiencias creativas de los participantes durante concursos o acciones de creación genera una experiencia basada en el interés, en factores económicos o en la competencia y habilidad de cada usuario. Los consumidores manifiestan intenciones propias para participar o aportar en entornos digitales, lo cual ejerce una influencia directa sobre cómo será su experiencia posterior. También, factores como la actitud, la utilidad percibida o la intención de participación o aportación online son determinantes para decidir formar parte de acciones de participación (Casaló, Flavián y Guinalíu, 2010), como en el caso de las acciones de cocreación textil.

La cocreación online supone una estrategia de innovación que contribuye de forma relevante a la experiencia del usuario, ya que el consumidor es capaz de realizar acciones de aportación entre diversos agentes y bajo diferentes perspectivas: diálogo continuo, acceso a la información, equilibrio riesgo/beneficio o transparencia de información (Prahalad y Ramaswamy, 2004). Autores como Hoyer et al. (2010) indican que las acciones de cocreación son una nueva perspectiva en relación al productor y al consumidor, ya que permiten la participación directa de los clientes en un marco de aportación de valor, pero enfocado después en su experiencia. La experiencia de cocreación de cada individuo dependerá de múltiples factores, como el grado de implicación del usuario, la valoración por parte de la marca durante la realización de la actividad, el beneficio percibido por participar, etcétera. Mientras las experiencias personales se enfocan normalmente en la observación, el aprendizaje o la participación en sucesos concretos que conectan al individuo con ese recuerdo, la experiencia basada en los consumidores de un mercado como el de la industria textil contempla una perspectiva mucho más extensa y está condicionada por diversos factores. Algunos autores (Mathis, Kim, Uysal, Sirgy y Prebensen, 2016) indican, en relación a la experiencia de cocreación del individuo, que el concepto de experiencia del usuario en cocreación está en relación con diversas dimensiones que afectan al comportamiento, al bienestar subjetivo o a la lealtad. Otros autores (Prebensen, Kim y Uysal, 2016) identifican la experiencia en cocreación según la relación entre el valor, la participación y la satisfacción percibida, indicando que cuanto más alto sea el nivel de participación en la acción de cocreación, más fuerte será el vínculo entre experiencia y satisfacción percibidas, y viceversa. Para Verleye (2015), la experiencia de cocreación se basa en diferentes dimensiones que la guían (hedónica, cognitiva, social, personal, pragmática y económica) y que, por tanto, la plantean como un fenómeno multidimensional. Verleye (2015) expone que la experiencia de cocreación no solo está influenciada por el proceso de cocreación en sí, sino también por entornos próximos a la cocreación (tecnológicos, de interacción, etcétera). Esta perspectiva es aplicable de forma integral a la cocreación online en el sector textil. Para otros autores (Jaakkola, Helkkula y Aarikka-Strenroos, 2015), la experiencia de cocreación se conecta de forma directa con elementos externos como el valor, la sociabilidad en los actuales mercados o los métodos de medición para gestionar servicios y experiencias. Por otra parte, la experiencia del consumidor online también puede estar ampliamente condicionada por aspectos como el eWom, estableciendo intercambio de información a través de Internet sobre un producto o servicio que pertenece a una empresa (Chu y Kim, $\triangleright$ 
2011) o condicionando la percepción del consumidor online hacia productos o marcas textiles por la recepción de diversas opiniones, valoraciones o críticas por parte de la experiencia de otros usuarios. Igualmente, es una vía de comunicación para que el consumidor traslade su experiencia a otros usuarios, una vez la haya experimentado. En definitiva, la experiencia de los usuarios online con las acciones de cocreación es clave para establecer plataformas cada vez más innovadoras y efectivas (Kohler, Fueller, Matzler y Stieger, 2011).

\section{Conclusiones}

A través de la revisión bibliográfica expuesta en este trabajo se pretende identificar la importancia que suponen las acciones de cocreación online en el sector textil y en la industria de la moda, como ámbitos de creación, innovación y desarrollo. La evolución del sector textil en España deriva de forma progresiva hacia estrategias online más centradas en la participación conjunta entre clientes y marcas textiles. La importancia de poder utilizar herramientas online, capaces de interactuar con los consumidores de moda de forma personalizada, permite a las empresas textiles obtener valiosa información sobre preferencias de consumo, necesidades, opiniones o sugerencias del mercado, A través de las acciones de cocreación online en moda es posible redirigir la creación o configuración de los productos textiles de una forma más amplia, innovadora y externalizada, contando con la participación activa de los consumidores y aportando valor de forma conjunta entre marcas y consumidores. Las empresas del sector textil en España aplican ya, en los procesos de creación de muchos productos, acciones de cocreación o participación con sus clientes que les aportan relevantes beneficios respecto a futuros planteamientos empresariales. De igual forma, los consumidores obtienen diversos grados de experiencia gracias a su participación en acciones de cocreación online en el sector textil, la cual puede estar motivada por diferentes factores, como el intercambio social, el aprendizaje, la interacción con la marca textil o la recompensa económica. Además, los actuales consumidores online en el sector de la moda desempeñan un papel mucho más activo y decisivo, siendo agentes altamente influyentes y compartiendo su propia experiencia de cocreación online con otros consumidores o sus preferencias $u$ opiniones sobre marcas y productos textiles, basadas en enfoques de participación activa a través de Internet.

\section{Bibliografía}

[1] AHEARNE, M.; BHATTACHARYA, C.B. y GRUEN, T. (2005). «Antecedents and Consequences of Customer-Company Identification: Expanding the Role of Relationship Marketing”. Journal of Applied Psychology, vol. 90, n. 3 , pp. 574-585.

[2] BALLANTYNE, D. y VAREY, R.J. (2008). «The service-dominant logic and the future of Marketing". Journal of the Academy of Marketing Science, vol. 36, n.1, pp. 11-14.

[3] BASON, C. (2010). Leading Public Sector Innovation: co-creating for a better society. Bristol, UK: The Policy Press Editorial.

[4] BETTENCOURT, L.A.; LUSCH, R.F. y VARGO, S.L. (2014). «A service lens on value creation: Marketing's role in achieving strategic advantage». California Management Review, vol. 57, n. 1, pp. 44-66.

[5] BLÁZQUEZ, M. (2014). «Fashion shopping in multichannel retail: The role of technology in enhancing the customer experience». International Journal of Electronic Commerce, vol. 18, n. -4 , pp. $97-116$. 
[6] CARLSON, J.; O'CASS, A. y AHRHOLDT, D. (2015). «Assessing customers' perceived value of the online channel of multichannel retailers: A two country examination». Journal of Retailing and Consumer Services, n.․ 27, pp. 90-102.

[7] CASALÓ, L. V.; FLAVIÁN, C. y GUINALÍU, M. (2010). «Determinants of the intention to participate in firm-hosted online travel communities and effects on consumer behavioural intentions». Tourism Management, vol. 31, n. .96 , pp. 898-911.

[8] CONSTANTINIDES, E.; BRÜNINK, L.A. y LORENZO-ROMERO, L.A. (2015). "Customer motives and benefits for participating in online co-creation activities». Internet Marketing and Advertising, vol. 9, n.․ 1, pp. 21-47.

[9] CHAE, H. y KO, E. (2016). «Customer social participation in the social networking services and its impact upon the customer equity of global fashion brands". Journal of Business Research, vol. 69, n. 9, pp. 3804-3812.

[10] CHAE, H.; KO, E. y HAN, J. (2015). «How do customers' SNS participation activities impact on customer equity drivers and customer loyalty? Focus on the SNS services of a global SPA brand". Journal of Global Scholars of Marketing Science, vol. 25, $\mathrm{n} .-\mathrm{o}$, pp. 122-141.

[11] CHEN, Y.; SHANG, R. y KAO, CH. (2009). The effects of information overload on consumers' subjective state towards buying decision in the internet shopping environment. Electronic Commerce Research and Applications, vol. 8, n. ${ }^{-1}$, pp. $48-58$.

[12] CHU, S.C. y KIM, Y. (2011). "Determinants of consumer engagement in electronic wordof-mouth (eWOM) in social networking sites". International Journal of Advertising, vol. 30, n. .1 , pp. 47-75.

[13] CNMC (2018). Ventas online de moda en España entre 2012-2017. Disponible en: https://www.modaes.es/files/000_2016/0001publicaciones/pdfs/informe_ecommerce_2018. pdf [Recuperado: 2018, 28 de marzo].

[14] DAMANPOUR, F. y GOPALAKRISHNAN, S. (2001). «The dynamics of the adoption of products and process innovations in organizations». Journal of Management Studies, ก.. 38, pp. 45-65.
[15] DEL OLMO, J.L. y GASCÓN, J.F.F. (2014). Marketing digital en la moda. Navarra: Ediciones Universidad de Navarra.

[16] DEL PINO, C.; GALÁN, E.; CASTELLÓ, A. y RAMOS, I. (2014). «Twitter como canal de comunicación corporativa y publicitaria». Communication \& Society, n.․ 27, pp. 21-54.

[17] EROGLU, S.; MACHLEIT, K. y DAVIS, L. (2003). «Empirical testing of a model of online store atmospherics and shopper responses". Psychology \& Marketing, vol. 20, n. .2 , pp. 139-150.

[18] FUCHS, C. y SCHREIER, M. (2011). "Customer Empowerment in New Product Development». Journal of Product Innovation Management, n.을, pp. 17-32.

[19] FÜLLER, J.; HUTTER, K. y FAULLANT, R. (2011). «Why co-creation experience matters? Creative experience and its impact on the quantity and quality of creative contributions". R\&D Management, vol. 41, n.ㅇ 3, pp. 259-273.

[20] GAMBETTI, R. C. y GRAFFIGNA, G. (2011). The concept of engagement. A systematic analysis of the ongoing marketing. Madrid: Editorial Debate.

[21] GOODMAN, S. y REMAUD, H. (2015). "Store choice: How understanding consumer choice of where to shop may assist the small retailer». Journal of Retailing and Consumer Services, n.. 23, pp. 118-124.

[22] GRANATA, F. (2012). "Fashion Studies Inbetween: A Methodological Case Study and an Inquiry into the State of Fashion Studies». Fashion Theory, vol. 16, n. 1, pp. 67-82.

[23] GRÖNROOS, C.; STRANDVIK, T. y HEINONEN, K. (2015). «Value Co-Creation: Critical Reflections». The Nordic School: Service Marketing and Management for the Future, Hanken: School of Economics, pp. 69-81.

[24] GRÖNROOS, C. y VOIMA, P. (2013). «Critical service logic: making sense of value creation and co- creation». Journal of the Academy of Marketing Science, vol. 41, n.으. 2, pp. 133-150.

[25] GRUNER, R.L.; HOMBURG, C. y LUKAS, B.A. (2014). «Firm-hosted online brand communities and new product success». Journal of the Academy of Marketing Science, vol. 42, n. ${ }^{\circ}$ 1, pp. 29-48.

[26] GUMMESSON, E. y MELE, C. (2010). «Marketing as Value Co-creation through Network $D$ 
Interaction and Resource Integration». Journal of Business Market Management, vol. 4, n. 4 , pp. 181-198.

[27] HEINONEN, K. y STRANDVIK, T. (2015). «Customer-dominant logic: foundations and implications». Journal of Services Marketing, vol. 29, n. .9 , pp. 472-484.

[28] HINZ, O.; SKIERA, B.; BARROT, C. y BECKER, J.U. (2011). «Seeding strategies for viral marketing: an empirical comparison». Journal of Marketing, vol. 75, n.ㅇ 6, pp. 55-71.

[29] HOLLEBEEK, L. D. (2011). «Demystifying customer brand engagement: exploring the loyalty nexus". Journal of Marketing Management, vol. 27, n. - 7-8, pp. 785-807.

[30] HOSANY, S. y WITMAN, M. (2010). «Dimensions of Cruisers Experiences. Satisfaction and Intention to Recommend». Journal of Travel Research, n. -9 49, pp. 351-364.

[31] HOYER, W.; CHANDY, R.; DOROTIC, M.; KRAFFT, M. y SINGH, S.S. (2010). «Consumer Cocreation in New Product Development». Journal of Service Research, n.. 13, n.. 3, pp. 283-296.

[32] HU, H.H.; LIN, J.; QIAN, Y. y SUN, J. (2018). «Strategies for new product diffusion: Whom and how to target?». Journal of Business Research, n.. 83, pp. 111-119.

[33] HULL, F. (2004). «Innovation strategy and the impact of a composite model of service product development on performance». Journal of Service Research, vol. 7, n.을, pp. 167-180.

[34] IAB SPAIN (2017). Estudio anual sobre e-Commerce 2017. Elaborado por IAB Spain y Elogia. Disponible en: https://iabspain.es/ wp-content/uploads/estudio-ecommerceiab-2017_vpublica.pdf [Recuperado: 2018, 3 de marzo].

[35] IM, S.; MONTOYA, M. y WORKMAN, J. (2012). «Antecedents and consequences of creativity in product innovation teams". Journal of Product Innovation Management, vol. 30, n.. 1, pp. $170-185$.

[36] IND, N. y COATES, N. (2013). «The meanings of co-creation». European Business Review, vol. 25, n. .9 1, pp. 86-95.

[37] INFORME DE LA MODA ONLINE EN ESPAÑA (2018). Elaborado por moda.es, Kantar Worldpanel y commerce cloud. Disponible en: https://www.modaes.es/files/000_2016/ 0001 publicaciones/pdfs/informe_ecommerce 2018.pdf [Recuperado: 2018, 15 de abril].

[38] JAAKKOLA, E.; HELKKULA, A. y AARIKKASTENROOS, L. (2015). «Service experience co-creation: conceptualization, implications, and future research directions». Journal of Service Management, vol. 26, n.ㅇ 2, pp. 182-205.

[39] JACKSON, T. y SHAW, D. (2006). The Fashion Handbook. Londres y Nueva York: Routledge.

[40] KASOF, J. (1995). «Explaining creativity: The attributional perspective». Creativity Research Journal, vol. 8, n.ㅇ 4, pp. 311-366.

[41] KIM, A.J. y KO, E. (2012). «Do social media marketing activities enhance customer equity? An empirical study of luxury fashion brand». Journal of Business Research, vol. 65, n. -10 , pp. 1480-1486.

[42] KOHLER, T.; FUELLER, J.; MATZLER, K. y STIEGER, D. (2011). "Co-creation in Virtual Worlds: The Design of the User Experience». MIS Quarterly, vol. 35, n. 3, pp. 773-788.

[43] KURUK, C. (2008). «Fashion as Communication: a Semiotic Analysis of Fashion on Sex and the City». Semiotica, vol. 171, n. .01 , pp. 193-214.

[44] LEEFLANG, P.S.; VERHOEF, P.C.; DAHLSTRÖM, P.y FREUNDT, T. (2014). «Challenges and solutions for marketing in a digital era". European Management Journal, vol. 32, n. 1 , pp. 1-12.

[45] LEMON, K.N. y VERHOEF, P.C. (2016). «Understanding customer experience throughout the customer journey». Journal of Marketing, vol. 80, n. .6 , pp. 69-96.

[46] LLORENTE, C.; BARTOLOMÉ, A. y NAVARRO, E. (2013). «Eficacia publicitaria en redes sociales: el caso de Mango en Facebook España». Questiones Publicitarias, vol. 1, n.․ 18, pp. 93-110.

[47] MAGNUSSON, P.R.; MATTHING, J. y KRISTENSSON, P. (2003). «Managing user involvement in service innovation: Experiments with innovating end users». Journal of Service Research, vol. 6, n.으. 2, pp. 111-124.

[48] MARUYAMA, M. y WU, L. (2014). «Multiple store patronage: the effects of store characteristics». Journal of Retailing and Consumer Services, vol. 21, n.ำ 4, pp. 601-609. 
Carlota Lorenzo Romero, Juan Antonio Mondéjar Jiménez y Leticia del Pozo Ruiz

[49] MATHIS, E.F.; KIM, H.L.; UYSAL, M.; SIRGY, J.M. y PREBENSEN, N.K. (2016). «The effect of co-creation experience on outcome variable». Annals of Tourism Research, n. 57 , pp. 62-75.

[50] McNEIL, P. (2010). «Conference Report: The Future of Fashion Studies». Fashion Theory, vol. 14, n.․ 1, pp. 105-110.

[51] NAMBISAN, S. y BARON, R.A. (2009). «Virtual Customer Environments: Testing a Model of Voluntary Participation in Value Co-creation». Journal of Product Innovation Management, n. -26 , pp. 388-406.

[52] NARANJO-VALENCIA, J.C.; JIMÉNEZ-JIMÉNEZ, D. y SANZ-VALLE, R. (2016). «Studying the links between organizational culture, innovation, and performance in Spanish companies». Revista Latinoamericana de Psicología, vol. 48, n. .9 , pp. 30-41.

[53] NEGHINA, C.; CANIËLS, M.; BLOEMER, J. y VAN BIRGELEN, M. (2015). «Value co-creation in service interactions Dimensions and antecedents». Marketing Theory, vol. 15, n.․․ 2, pp. 221-242.

[54] O'HERN, M.S. y RINDFLEISCH, A. (2010). "Customer co-creation». Review of Marketing Research, $n .{ }^{\circ} 6$, pp. 84-106.

[55] ORDANINI, A.; MICELI, L.; PIZZETTI, M. y PARASURAMAN, A. (2011). "Crowd-funding: transforming customers into investors through innovative service platforms". Journal of Service Management, vol. 11, n. 3 , pp. 443-470.

[56] PAYNE, A.F.; STORBACKA, K. y FROW, P. (2008). Managing the co-creation of value. Journal of the Academy of Marketing Science, vol. 36, n.․ 1, pp. 83-96.

[57] PEREIRA, P.A.; GARCÍA I.; GONZÁLEZ, Z.F. y CONTRERAS-ESPINOSA, R.S. (2014). «The importance of Facebook as an online social networking tool for companies». International Journal of Accounting and Information Management, vol. 22, n.․ 4, pp. 295-320.

[58] PRAHALAD, C.K. y RAMASWAMY, V. (2000). "Co-opting customer competence». Harvard Business Review, vol. 78, n.․ 1, pp. 79-87.

[59] PRAHALAD, C.K. y RAMASWAMY, V. (2004). "Co-creation experiences: The next practice in value creation". Journal of Interactive Marketing, vol. 18, n. 3, pp. 5-14.

[60] PREBENSEN, N.K.; KIM, H. y UYSAL, M. (2016). «Cocreation as moderator between the experience value and satisfaction relationship. Journal of Travel Research, vol. 55,

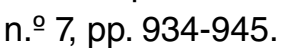

[61] RAMASWAMY, V. y GOUILLART, F. J. (2010). The power of co-creation: Build it with them to boost growth, productivity, and profits. Nueva York: Simon and Schuster Editorial.

[62] ROSER, T.; DEFILLIPPI, R. y SAMSON, A. (2013). "Managing your co-creation mix: co-creation ventures in distinctive contexts". European Business Review, vol. 25, n. -1 , pp. 20-41.

[63] RUBERA, G.; ORDANINI, A. y MAZURSKY, D. (2010). «Toward a contingency view of new product creativity: Assessing the interactive effects of consumers' characteristics". Marketing Letters, vol. 21, n.․ 2, pp. 191-206.

[64] SAARIJÄRVI, H.; KANNAN, P. y KUUSELA, H. (2013). «Value co-creation: theoretical approaches and practical implications». European Business Review, vol. 25, n. 1, pp. 6-19.

[65] SÁDABA, T. y SAN MIGUEL, P. (2014). «Revisión de los influentials en moda con la aparición de Internet: estudio del caso español a través de Stylelovely.com. Re-defining Fashion Influentials in the Internet Age: a Spanish Case-Study based». Revista de Comunicación, vol. 13, n.․ 61, pp. 60-83.

[66] SÁNCHEZ, J.A.L.; MIERES, C.G. y VIJANDE, M.L.S. (2013). «Innovación de servicio y co-creación con los clientes de la empresa: efectos sobre los resultados». Revista Española de Investigación en Marketing ESIC, vol. 17, n.. 2, pp. 79-102.

[67] SAWHNEY, M.; VERONA, G. y PRANDELLI, E. (2005). "Collaborating to create: The internet as a platform for customer engagement in product innovation». Journal of Interactive Marketing, vol. 19, n.․ 4, pp. 4-17.

[68] SICILIA, M. y RUIZ, S. (2010). The effects of the amount of information on cognitive responses in online purchasing tasks. Electronic Commerce Research and Applications, vol. 9, n. $\mathrm{o}$ 2, pp. 183-191.

[69] TORREGROSA, M.; NOGUERA, M.; SÁNCHEZ-BLANCO, C. y DEL RÍO, J. (2014). «La moda en España ante la convergencia de Bolonia. Un estudio desde la perspectiva de la comunicación». Sphera Publica, vol. 1, n. .14 , pp. 124-141. 
[70] TSEËLON, E. (2010). «Outlining a Fashion Studies Project». Critical Studies in Fashion and Beauty, vol. 1, n.․ 1, pp. 3-53.

[71] TSIOTSOU, R. y RATTEN, V. (2010). «Future research directions in tourism Marketing". Marketing Intelligence and Planning, vol. 28, n. -9 , pp. 533-544.

[72] VALLEJO, J.; REDONDO, Y. y ACERETE, A. (2015). «Las características del boca-oído electrónico y su influencia en la intención de recompra online. «Electronic word-of-mouth (eWom) characteristics and his influence in online repurchase intention». Revista Europea de Dirección y Economía de la Empresa, n.․ 24, pp. 61-75.

[73] VARADARAJAN, R. y YADAV, M. (2005). «Understanding product migration to the electronic marketplace: A conceptual framework». Journal of Retailing, vol. 81, n.․ 2, pp. 125-140.

[74] VARGO, S.L. y LUSCH, R.F. (2004). «Evolving to a new dominant logic for marketing". Journal of Marketing, vol. 68, n. 1, pp. 1-17.

[75] VARGO, S.L. y LUSCH, R.F (2011). «It's all B2B... and beyond: Toward a systems perspective of the market». Industrial Marketing Management, vol. 40, n.․ 2, pp. 181-187.

[76] VEGA, M.; REVILLA, M. y COSSíO, F. (2013). «The value co-creation process as a determinant of customer satisfaction». Management Decision, vol. 51, n. 19, pp. 1945-1953.

[77] VERLEYE, K. (2015). «The co-creation experience from the customer perspective: its measurement and determinants». Journal of Service Management, vol. 26, n.ㅇ 2, pp. 321-342.

[78] VON HIPPEL, E. (2005). Democratizing Innovation. Cambridge, Massachusetts: MIT Press Editorial.

[79] WASKO, M.M. y FARAJ, S. (2000). «It is What One Does: Why People Participate and Help Others in Electronic Communities of Practice». Journal of Strategic Information Systems, vol. 9, n.을 -3, pp. 155-173.

[80] YI, Y. y GONG, T. (2013). «Customer value co-creation behaviour: scale development and validation». Journal of Business Research, vol. 66, n. $\circ 9$, pp. 1279-1284. 

ISSN 0214-8307

\section{SUSCRIPCIÓN ANUAL}

\begin{tabular}{|c|c|c|c|}
\hline \multicolumn{3}{|c|}{ BOLETÍN ECONÓMICO DE INFORMACIÓN COMERCIAL ESPAÑOLA (12 NÚMEROS) } \\
\hline & $\begin{array}{c}\text { ESPAÑA } \\
\mathbf{1} \text { año }\end{array}$ & $\begin{array}{c}\text { UNIÓN EUROPEA } \\
\mathbf{1} \text { año }\end{array}$ & $\begin{array}{c}\text { RESTO DEL } \\
\text { MUNDO } \\
1 \text { año }\end{array}$ \\
\hline SUSCRIPCIÓN & $65,00 €$ & $85,00 €$ & $85,00 €$ \\
\hline $\begin{array}{c}\text { Gastos de envío } \\
\text { España }\end{array}$ & $5,76 €$ & $24,36 €$ & $30,00 €$ \\
\hline $\begin{array}{c}\text { Más 4\% de IVA. } \\
\text { Excepto Canarias, Ceuta y Melilla }\end{array}$ & $2,83 €$ & & $115,00 €$ \\
\hline TOTAL & $73,59 €$ & $109,36 €$ & \\
\hline & & & \\
\hline
\end{tabular}

\section{EJEMPLARES SUELTOS}

\begin{tabular}{|c|c|c|c|}
\hline BOLETÍN ECONÓMICO & DE INFORMACIÓN & COMERCIAL ESPAÑOL & \\
\hline & $\begin{array}{l}\text { ESPAÑA } \\
1 \text { ejemplar }\end{array}$ & $\begin{array}{l}\text { UNIÓN EUROPEA } \\
1 \text { ejemplar }\end{array}$ & $\begin{array}{l}\text { RESTO DEL } \\
\text { MUNDO } \\
1 \text { ejemplar }\end{array}$ \\
\hline NÚMERO SUELTO & $7,00 €$ & $9,00 €$ & $9,00 €$ \\
\hline $\begin{array}{l}\text { Gastos de envío } \\
\text { España }\end{array}$ & $0,48 €$ & $2,03 €$ & $2,50 €$ \\
\hline $\begin{array}{l}\text { Más } 4 \% \text { de IVA. } \\
\text { Excepto Canarias, Ceuta y Melilla }\end{array}$ & $0,30 €$ & & \\
\hline TOTAL & $7,78 €$ & $11,03 €$ & $11,50 €$ \\
\hline \multirow[t]{2}{*}{ BOLETÍN ECONÓMICO } & DE INFORMACIÓN & COMERCIAL ESPAÑOL & \\
\hline & $\begin{array}{l}\text { ESPAÑA } \\
1 \text { ejemplar }\end{array}$ & $\begin{array}{l}\text { UNIÓN EUROPEA } \\
1 \text { ejemplar }\end{array}$ & $\begin{array}{c}\text { RESTO DEL } \\
\text { MUNDO } \\
1 \text { ejemplar }\end{array}$ \\
\hline $\begin{array}{l}\text { NÚMERO SUELTO } \\
\text { EXTRAORDINARIO }\end{array}$ & $12,00 €$ & $15,00 €$ & $15,00 €$ \\
\hline Gastos de envío España & $0,48 €$ & $2,03 €$ & $2,50 €$ \\
\hline $\begin{array}{c}\text { Más 4\% de IVA. } \\
\text { Excepto Canarias, Ceuta y Melilla }\end{array}$ & $0,50 €$ & & \\
\hline TOTAL & $12,98 €$ & $17,03 €$ & $17,50 €$ \\
\hline
\end{tabular}

\section{DATOS}

Nombre y apellidos

Empresa

Domicilio

D.P.

N.I.F.

Teléf.

Email

\section{DATOS DEL EDITOR:}

NIF:S2800568D

Transferencia a la cuenta de ingresos por venta de publicaciones del Ministerio de Economía y Competitividad.

IBERCAJA. Calle Alcalá 29. 28014 MADRID (ESPAÑA) CÓDIGO CUENTA CLIENTE: 2085-9252-07-0330598330 CÓDIGO BIC DE IBERCAJA: CAZRES2Z

IBAN: ES47 2085-9252-07-0330598330

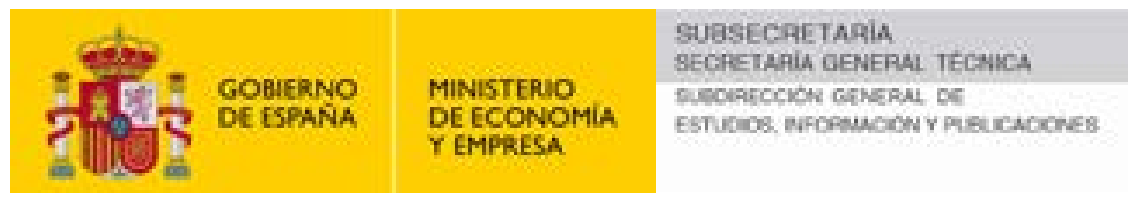

Suscripciones y ventas por correspondencia:

Paseo de la Castellana, $1628^{\text {a }}$ Planta 28046 Madrid. Teléfono 916037993 / 97

Suscripciones a través de la página web del Ministerio de Industria, Comercio y Turismo distribucionpublicaciones@mineco.es 


\section{NORMAS DE ESTILO DE PUBLICACIÓN}

La persona o personas interesadas en presentar un artículo para su publicación en el Boletín de Información Comercial Española (BICE) deberán enviar el artículo en formato Microsoft Word a la dirección de correo electrónico revistasice.sscc@comercio.mineco.es

El documento debe cumplir las siguientes características:

1. Ser material original no publicado ni presentado en otro medio de difusión.

2. La extensión total del trabajo (incluyendo cuadros, gráficos, tablas, notas, etcétera) no debe ser inferior a 15 páginas ni superior a 20. La fuente será Times New Roman, tamaño 12 y espaciado doble. Estar paginado en la parte inferior derecha de cada página.

3. En la primera página se hará constar el título del artículo que deberá ser breve, claro, preciso e informativo y la fecha de conclusión del mismo. Nombre y dos apellidos del autor o autores, filiación institucional, dirección, teléfono y correo electrónico de cada uno de ellos, así como la forma en que desean que sus datos aparezcan.

4. En la segunda página del texto se incluirá:

- El título.

- Un resumen del trabajo con una extensión máxima de 10 líneas con la siguiente estructura: objetivo, método y principal resultado o conclusión.

- De 2 a 6 palabras clave que no sean coincidentes con el título.

- De 1 a 5 códigos de materias del Journal of Economic Literature (clasificación JEL) para lo cual pueden acceder a la siguiente dirección electrónica:

https://www.aeaweb.org/jel/guide/jel.php

5. En las siguientes páginas se incluirán el texto, la información gráfica y la bibliografía con la siguiente organización.

- Se incluirá, por este orden, introducción, desarrollo, conclusiones y bibliografía y anexos si los hubiera. Los apartados y subapartados se numerarán en arábigos respondiendo a una sucesión continuada utilizando un punto para separar los niveles de división, según el siguiente modelo:

\section{Título del apartado}

\subsection{Título del apartado}

1.1.1. Título del apartado

\section{Título del apartado}

- Las notas de pie de página irán integradas en el texto y su contenido debe estar al final de su misma página en tamaño 10 y espacio sencillo.

- En un archivo Excel independiente se incluirá la representación gráfica (cuadros, gráficos, diagramas, figuras, etc.), que debe llevar título, estar numerada y referenciada en el texto. En la parte inferior se incluirá la fuente de información y/o notas aclaratorias.

- Las citas de libros y artículos en el texto, se indicarán entre paréntesis con el apellido del autor y el año. Ej.: (Martínez, 1991).

- Las referencias a siglas deben ir acompañadas, en la primera ocasión en que se citen, de su significado completo.

- La bibliografía se ordenará alfabéticamente siguiendo las normas de la American Psychological Association (Harvard-APA): http://cibem.org/paginas/img/apa6.pdf

Libros

APELLIDOS, A.A. (año de publicación). Título del libro (edición) (volumen). Ciudad: Editorial.

Artículo en revista científica

APELLIDOS, A.A. (año de publicación). «Título del artículo». Título de la revista, volumen (número), números de páginas.

Documento en línea

APELLIDOS, A.A. u ORGANISMO (año, mes de publicación). Título, [en línea]. Ciudad: Editorial. Disponible en:

http://cenamb.rect.ucv.ve/siamaz/dicciona/canaima/canaima2.htm [Recuperado: 2000, 3 de junio].

Los artículos publicados estarán disponibles en www.revistas/CE.com 
